\title{
Security architecture for the mobile ad hoc networks
}

\author{
Jozef Filipek, Ladislav Hudec ${ }^{*}$
}

\begin{abstract}
Security in mobile ad hoc networks (MANETs) has been an actively researched topic for the several years. As opposed to wired networks, MANETs have dynamic topology, limited resources, limited bandwidth and are usually deployed in emergency scenarios outside, where landscape plays important role. MANETs are susceptible to insider and outsider attacks and bring new security challenges which were not present in the wired networks. The most important difference is that every node in MANET acts as a router and routes traffic throughout the network. Compromising one node can hugely affect network performance. In this paper, we present our security architecture for MANETs which secures important aspects of the network. We bring trust model into the network and nodes are secured by different mechanisms tailored specifically for the use in distributed environment. We use Omnet++for network simulations. Simulations use delays measured on the real hardware and we analyze performance of the network during different data plane and control plane loads.
\end{abstract}

K e y w or ds: AES, attribute authority, architecture, firewall, MANET, PKI, RSA, security

\section{Introduction}

Advances in mobile devices such as higher computing power, low energy consumption and increasing internal storage make them more feasible to participate in the important roles in the network. Mobile devices were and still are usually used as the end devices but nowadays they are more and more capable to comprise internal part of the network.

MANETs are dynamic, self-configuring, mobile and easy to deploy devices. Comparing it to the wired networks, they do not need fixed infrastructure with the central point (router) and each MANET node expands networks reach and adds another computing resource into the network. However, almost every advantage these networks have over wired networks can be exploited and brings forth new security challenges that were present in wired network scenarios. Shared medium is susceptible to various threats. It can be easily eavesdropped and there are many ways to disrupt wireless communication. Disrupting at the physical layer is almost impossible to protect against and will not be in covered within our scope. Mobility of the nodes brings another security concern. It may divide the network if part of the nodes become unreachable and stranded nodes are easy target for the attack.

Since every node in the network acts like a router, behavior like that brings critical security challenge. What if one or more nodes gets compromised? What if some malicious outsider node gets connected into the network? Depending on the routing protocol used, one node can severely affect the way the network works. Since by default, MANETs do not use trust model, one compromised node can safely send malicious data to other nodes without any suspicion from other nodes.

In this paper, we will focus our security improvements onto control and data plane. We address several layers of security vulnerabilities, such as eavesdropping, behavior of the nodes, cryptography and dynamic trust model between nodes. More details will be in the following chapters.

\section{Related Work}

During our research, we encountered many papers focusing on the security of the MANETs. We were focusing our attention on IPS, Firewall and distributed security systems. Major categories of these works are: Intrusion Prevention Systems (IPS) [4] [6] [11] [13] [14] [20], Secured Routing Protocols [5] [8] [9] [12] [15] [17] [19] and Securing aspects of the MANETs, meaning, different mechanisms to protect networks against specific attacks, for example DoS (Denial of Service) or routing attacks. The least amount of papers was focused on the firewall systems because of the nature of the MANETs [1] [2] [3] [18] [21].

Based on our analysis of existing solutions and previous work with the security in MANETs, we decided to use existing PKI with secured routing solution developed in our department [16] and on top of it we are adding custom Firewall solution.

\section{Security Architecture}

As stated before, our solution consists of secured routing protocol along with PKI, Firewall and IPS. All components interoperate with each other and use the same

Faculty of Informatics and Information Technologies, Slovak University of Technology in Bratislava, Bratislava, Slovakia, xfilipekj1@stuba.sk, ladislav.hudec@stuba.sk 
sources of information. At the lowest layer, we are using secured routing protocol BATMAN along with PKI. On top of it, in terms of network layers, is our Firewall solution. IPS is enforcing policies amongst all nodes given by PKI and Firewall. With this architecture, we are trying to solve following vulnerabilities in MANETs:

- Trust between nodes

- Confidentiality and integrity of the communication

- Dynamic changes in nodes behavior

Based on our analysis, simulation and measurements, we decided to use hashing function SHA-512, asymmetric algorithm RSA with key length of $1024 \mathrm{~b}$ and symmetric algorithm AES with key lengths of 128 bits.

\subsection{Public Key Infrastructure with Secured Routing Protocol}

This is where we put integrity checks and trust model. Our approach uses secured version of BATMAN protocol and PKI model which communicates with the help of the routing protocol. It serves as a security underlay of our solution.

Trust model uses 4 level of privileges for nodes (L0 L3). L0 nodes are considered outsider nodes and are not trusted in any way. The only activity they are allowed to do, is to request the certificate from the AA (Attribute Authority). After receiving the certificate, they become L1 nodes. L1 nodes are allowed end-to-end communication within the network. However, as the precaution and security measure, they are not allowed to participate in the routing. That makes newcomers into the network less dangerous and for the attackers it is not feasible position to source the attack from. After some time and when nodes behavior complements with network policies, they can request raising their privileges to L2. L2 participate in the routing, distributed storage and IPS. Based on the abnormalities in the network, it can revoke certificates locally, i.e. does not allow communication of misbehaving nodes through itself and generate alarm about them, which goes to other L2 and L3 nodes. After enough time and clean policy history, L2 nodes may request elevating their privileges to L3. L3 is stand-alone AA and can certify other nodes. Every AA creates its own ecosystem, i.e. nodes that have certificates from said AA. Two AAs create 2 different ecosystems and for them to inter-operate with each other, they need to perform cross-certification. That way can ecosystems communicate with each other. BATMAN is modified and secured with RSA algorithm using public/private keys with the length of 1024 bits. AA is responsible for handing out attribute certificates to individual nodes. Certificates are signed by AA and every node can verify validity of the certificate with AAs public key. Certificates have limited validity and when their validity is about to expire, nodes have to ask their AA to recertify them. For this modification to work, BATMAN has new and modified control messages. Crt_request and crt_response for the request and response of the missing certificate from the node and BATMAN protocol message modified for the use of PKI and several ecosystems, Fig. 1 to 3 respectively.

\begin{tabular}{|c|c|}
\hline Packet type & Certificate hash \\
\hline \multicolumn{2}{|c|}{ Certificate hash } \\
\hline Certificate hash & Issuer hash \\
\hline \multicolumn{2}{|c|}{ Issuer hash } \\
\hline Issuer hash & Padding \\
\hline
\end{tabular}

Fig. 1. BATMAN crt_request control message

\begin{tabular}{|l|l|l|}
\hline Packet type & Data length & Certificate \\
\hline \multicolumn{3}{|c|}{ Certificate... } \\
\hline
\end{tabular}

Fig. 2. BATMAN crt_response control message

\begin{tabular}{|c|c|c|c|}
\hline Packet type & Version & TTL & TT Version \\
\hline \multicolumn{3}{|c|}{ Destination } \\
\hline \multicolumn{3}{|c|}{ CA_crt_hash } \\
\hline Destination & CA_crt_hash \\
\hline \multicolumn{3}{|c|}{ Encaps. Ethernet header } \\
\hline CA_crt_hash &
\end{tabular}

Fig. 3. BATMAN packet with encapsulated data

Every BATMAN message is signed by respective senders private key and for the successful processing of such message it is necessary for all nodes who are communicating with each other to have certificates necessary for signature verification.

\subsection{Firewall}

Firewall serves as a security overlay in our architecture. It brings confidentiality and data communication policy enforcement. How exactly performs its operations will be explained in this chapter. Firewall is usually placed at an entry point into the network and all communication between the outside and the inside of the network is supposed to go through it. In MANETs, it is next to impossible to utilize this concept, since every node routes traffic and even if we were to limit communication in the network through several handpicked nodes, it would not be very feasible solution due to the nature of MANETs. We decided to use different approach. Based on our previous work and simulations, we concluded our Firewall needs to satisfy several requirements:

- Initial deployment of the Firewall Policies

- Defined attributes and certificates

- Secured exchange of the shared secret for data confidentiality 


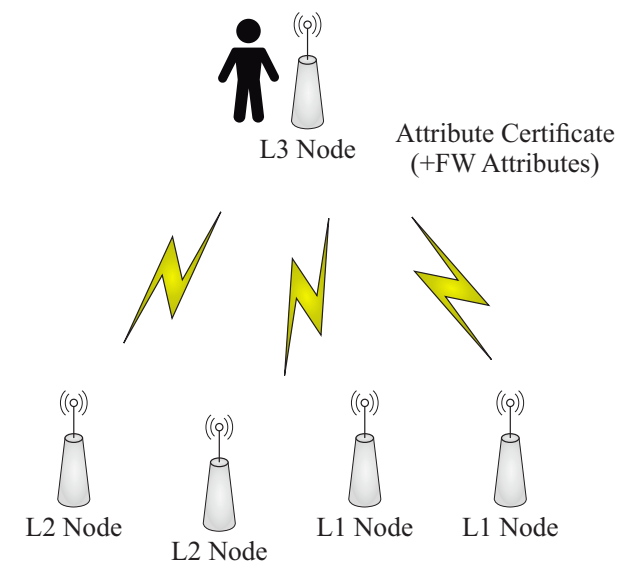

Fig. 4. Handing out certificates during creation of the network

- Storage of the used data

- Control messages required for the communication

- Communication model

\subsubsection{Initial deployment of the firewall policies}

Every node contains Firewall mechanism and can police other nodes, except L0 and L1 nodes. This is done via special attributes that can limit used bandwidth of the nodes, their communication radius and services they can use. Initial attributes are part of the AAs certificate. Every other node can see them and can police the communicating node based on their values. Figure 1 depicts handing out certificates by the AA. They contain also FW attributes needed for traffic policing. This is the only unprotected part of the communication in our architecture. It is vulnerable to eavesdropping and MITM (Man in the Middle) attacks. The only feasible way to protect this step in the communication would be do it in the controlled environment or preload nodes with the certificates.

\subsubsection{Defined attributes and certificates}

Attributes are defined in the attribute certificate of the node with following semantics:

Destination_FC: aa** / Communication radius

Service_FC: 3 / Allowed services

Bandwidth_FC: 3 / Maximum allowed bandwidth in multiplications of $64 \mathrm{kbps}$

Secondly, we needed some sort of more granular and dynamic control for other nodes. This is achieved via Session certificates. Their semantics below:

Serial_Number_SC: 130745 / Session ID

Issuer_SC: Aaaa / Certificate owner

Subject_SC: aaaa / Communication radius

Validity_SC: 2013-10-25 / Certificate validity

Service_SC: 3 / Allowed service

Bandwidth_SC: 10 / Allowed bandwidth

signature_SC: sig-rsa 23455656 / Signature

Certificate negotiation is described in the following chapter.

\subsubsection{Secured exchange of the shared secret for data confidentiality}

Allowed bandwidth in the attributes of the attribute certificate is sufficient for the creation of the session certificates. Session certificate is created when node wants to perform end-to-end communication with some other node in the network. Session certificates are based on the RSA and at this point in the communication, every node already has RSA key pair assigned. Figure 5 depicts establishment of the session certificates along with shared secret creation. We use DH algorithm to safely compute shared secret. Session certificates are valid only for the limited time and only for the selected pair of the nodes. During this exchange, nodes request more bandwidth for the subsequent data communication. As seen in the Figure 5 , transmitter may request more bandwidth than the receiver allows. Receiver has the final word and transmitter has to accept it decision. Decision is based on the current receivers utilization (CPU, memory and bandwidth).

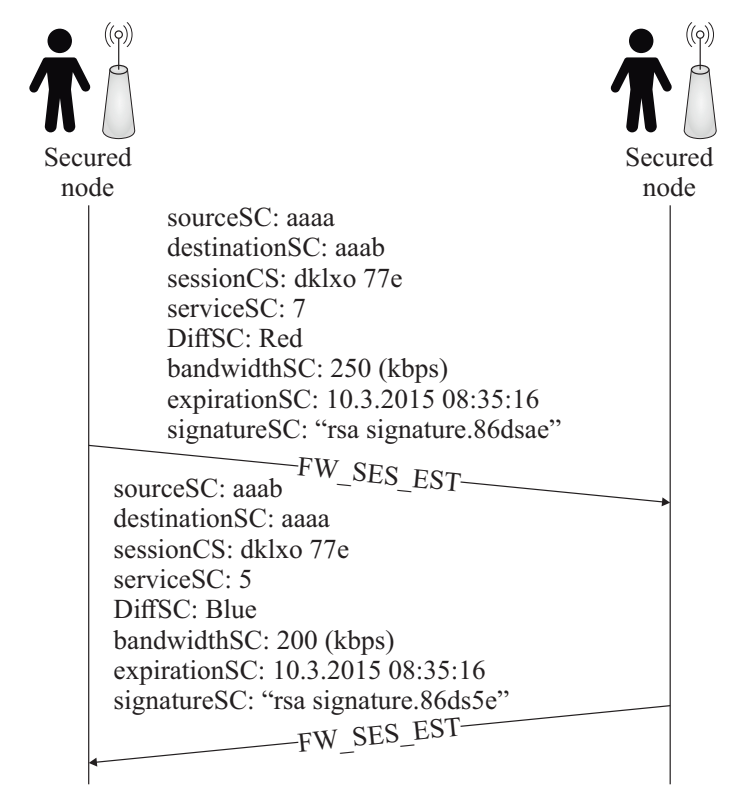

Fig. 5. Session certificate establishment

\subsubsection{Storage of the used data}

Whole architecture uses same databases for its functionality:

- Certificate database

- Violation database

Certificate database is used for storing attribute and session certificates. Every packet flowing thorough the node is cross-checked against this database.

Violation database contains information about nodes who in some way violated network policies. Either integrity check fails, sending too much traffic and so on. 
\begin{tabular}{|l|l|l|}
\hline Packet type (8b) & Certificate hash (512b) & Certificate (1kB) \\
\hline
\end{tabular}

Fig. 6. : $\mathrm{fw}_{s} e s_{e} s t / f w_{s} e s_{r}$ espacket

\begin{tabular}{|l|l|}
\hline Packet type (8b) & Certificate hash (256b) \\
\hline
\end{tabular}

Fig. 7. $\mathrm{FW}_{S} E S_{M}$ ISpacket

\begin{tabular}{|l|c|c|}
\hline Packet type (8b) & $\begin{array}{c}\text { Certificate hash } \\
\text { (256b) }\end{array}$ & Data \\
\hline
\end{tabular}

Fig. 8. $\mathrm{FW}_{D}$ AT Apacket

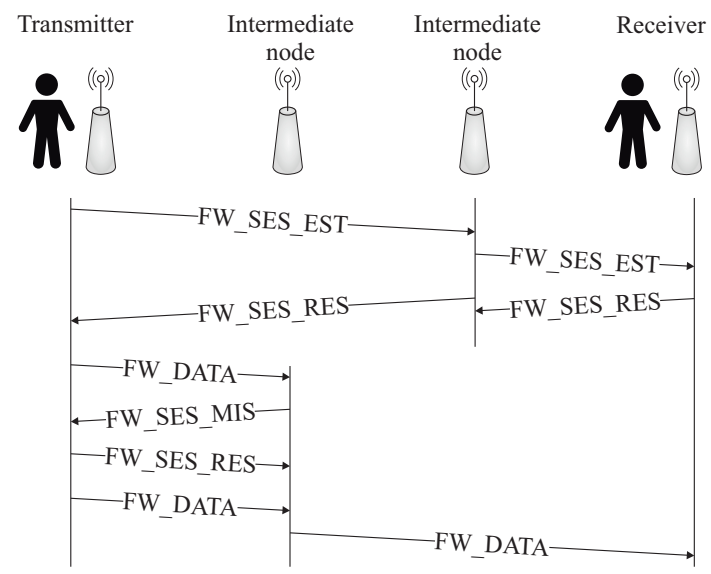

Fig. 9. Data communication

\subsubsection{Control messages required for the communication}

Based on our analysis and tests, Firewall portion of the architecture consists of four control messages that were needed for its smooth functionality.

FW_SES_EST (Firewall Session Establishment) is used in the process of creating session certificate, Figure 6 . Both sides use the same structure of the packet. Difference is only in the field Packet Type, where it can mean that the packet was sent either by a receiver or a transmitter.

FW_SES_MIS (Firewall Session Missing) is used when intermediate node is missing session certificate. This packet contains hash of the missing certificate and the signature of the entire packet, see Fig. 7.

FW_DATA (Firewall Data) is encapsulated data payload with the Firewall control information. The only identification is certificate hash of the Session certificate. Fig. 8 .

It is worthy to mention that Firewall layer does not perform any asymmetric cryptography. Its packets are already signed by lower level encapsulation performed by BATMAN.

\subsubsection{Communication model}

Figure 9 depicts data communication between nodes in the network. At first, there is Session Certificate exchange during which shared key will be computed. After that, nodes can safely send traffic between each other. We can see, that initial exchange is going through different intermediate node that subsequent communication, that may be caused by many factors, for example nodes could have moved or there was some change in the radio signal. When the new intermediate node does not know about session certificate communication nodes are using, it drops received traffic and requests certificate from the sending node. After receiving missing certificate, it can continue forwarding traffic between those nodes.

There are three scenarios as to how the Firewall handles communications:

- Receiving data from the upper layer

- Receiving data from the lower layer

- Session certificate handling (ie Receival, Sending and Processing)

When receiving data from the upper layer, existing sessions are checked. If there has already been created a session between nodes, node just encrypts the data and forwards it. In the case of completely new communication request, session certificate is created and sent to the communication receiver. Upon receipt of session certificate, initiator and receiver handle certificates differently. Receiver checks available resources and based on them decides whether he acknowledges Initiators requirements or has to modify them. Then he takes part in its own DH computation, creates session certificate and sends it to the Initiator. Initiator finishes DH computation and stores certificate in its database. Session certificate handling is depicted in Fig. 10.

\subsection{Intrusion prevention}

IPS is essential in our security architecture. It is controlling nodes and makes sure they adhere security policies. Security po icies consist of PKI and Firewall rules. Depending on the severity of the offense, nodes may have their certificates revoked completely or temporarily.

PKI with routing protocol takes care of signing communication and making sure it has not been tampered with during transmissions and Firewall is responsible for confidential transfer of data with the distribution of network constraints which can be enforced by every relevant node, ie L2 and L3. All alarms and events are propagated thanks to DHT (Distributed Hash Table).

\subsection{Security analysis}

From the security point of view, our architecture secures nodes at routing layer with asymmetric algorithm, data communication is secured at the network layer and behaviour of the nodes is being controlled by PKI, Firewall and IPS. Following are key points of the additional security our approach provides: 


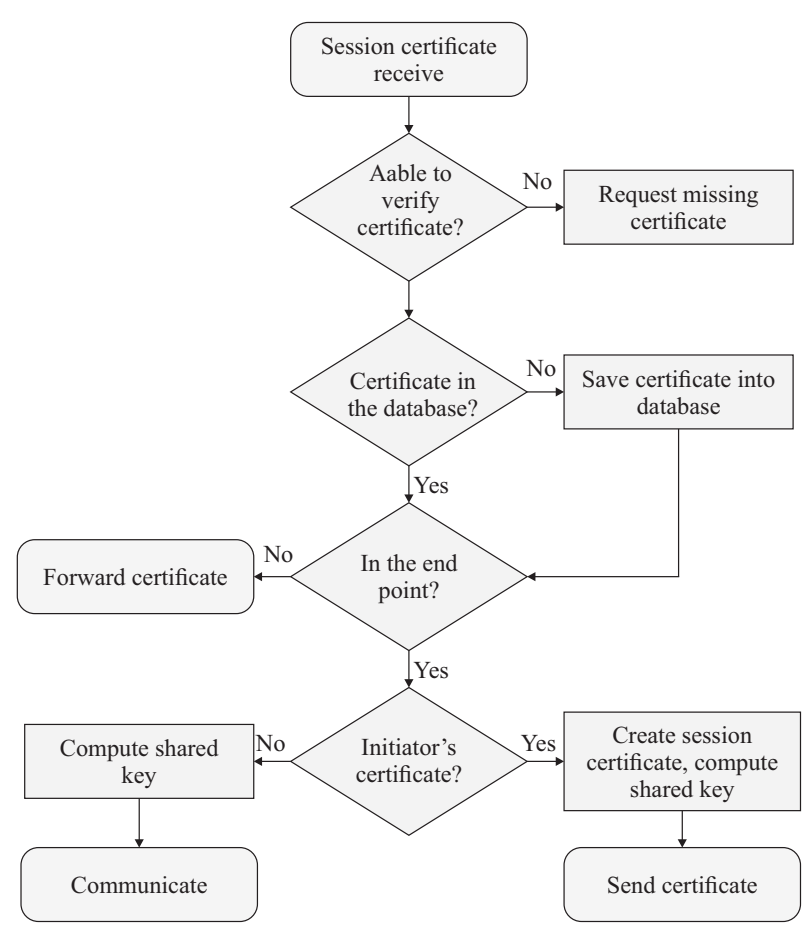

Fig. 10. Session certificate handling

- Signing and verifying communication with the RSA at the routing and data layer (due to BATMAN encapsulation data payload)

- Encrypting network layer information with symmetric AES algorithm

- Dividing nodes privileges into several groups

- New nodes are not participating in the essential parts of the network: Routing, Intrusion detection

- Delayed participation of nodes in important tasks mentioned above makes them unattractive for the attackers

- Dynamic changes in nodes privileges

- Higher level nodes (L2 and L3) police other nodes and based on their IPS alerts and DHT cooperation L3 can revoke certificate of the malicious nodes

- Communication of the unsecured nodes is denied by default

- Secured nodes are limited by their own privileges and Firewall constraints

The biggest threat in the MANETs is the compromising of the existing secured nodes. The higher privilege level, the bigger threat it can pose to the network. That is why nodes can become L3, AAs, so there would be another cross certification between ecosystems and in the case of failure of one AA another can take over.

\section{Simulation Results}

Security architecture was implemented in the Omnet ++ simulation environment. We used measurement of cryptographic operations on the real hardware to create base representation for our simulation. For these measurements, quad core ARM Cortex $900 \mathrm{MHz}$ processor was used, which has little worse performance than current average mobile devices. It performed 1363 RSA signs and 25994 RSA verifications within 10s time interval. AES performed at 63895 encryptions/decryptions in $3 \mathrm{~s}$.

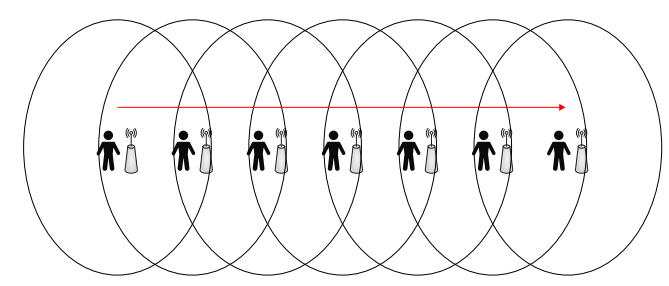

Fig. 11. Line topology

Following are representations of the simulations performed on the Line topology (Figure 11).

For the following measurement we used UDP CBR traffic. Packets had 1350B, sent every 0.04s. Sending started at the 7th second of the simulation. Figure 12 represents packet loss based on the number of passed hops. Loss with the secured architecture was higher than without it, which basically represents routing protocol effectivity, but it was still within acceptable limits.

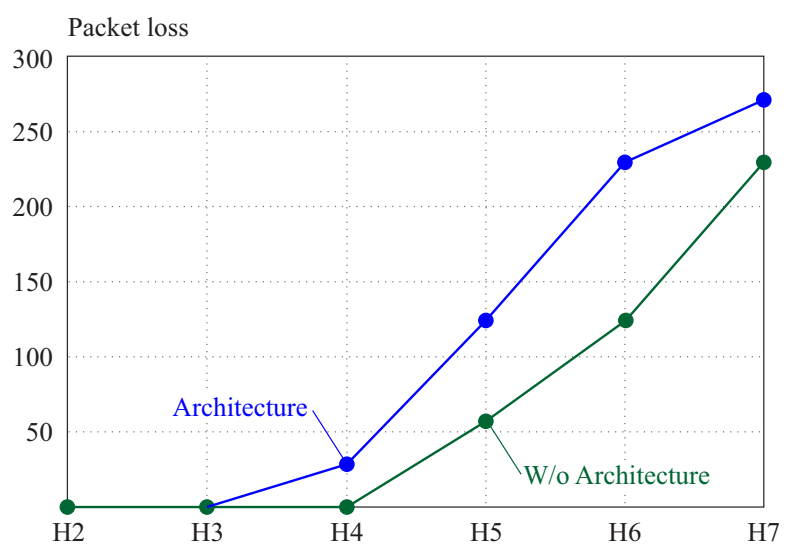

Fig. 12. Packet loss

Throughput of the UDP traffic is depicted in the Figure 13. Throughput was also lower based on the number of passed hops, but it was still within acceptable limits and symmetric encryption did not bring much strain on the available bandwidth.

Figure 14 shows latency based on the number of hops. Latency suffers most in ad hoc networks and we can clearly see that it is getting much worse with the increasing number of hops.

Figure 15 and Figure 16 show difference between network using our Architecture and without it using just plain routing protocol. We can see, that there is little difference caused by higher Architecture overhead and symetric data encryption. 


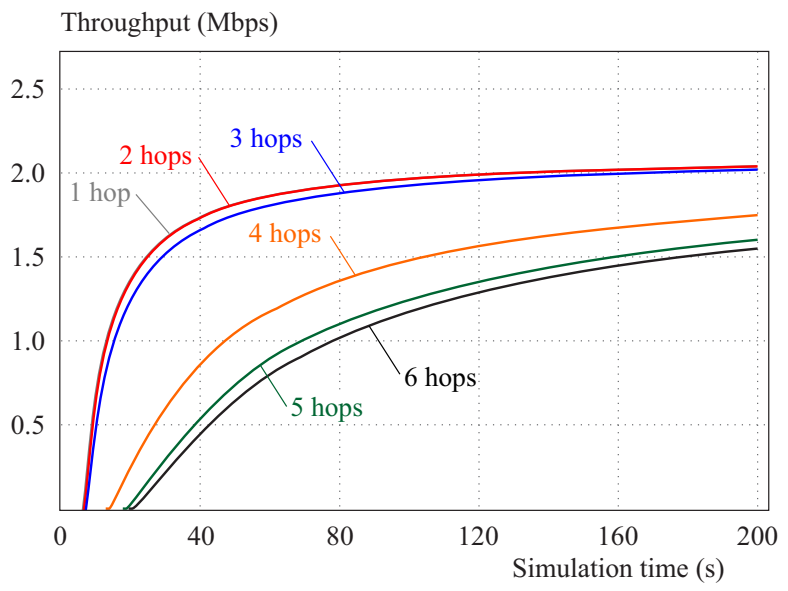

Fig. 13. Architecture throughput

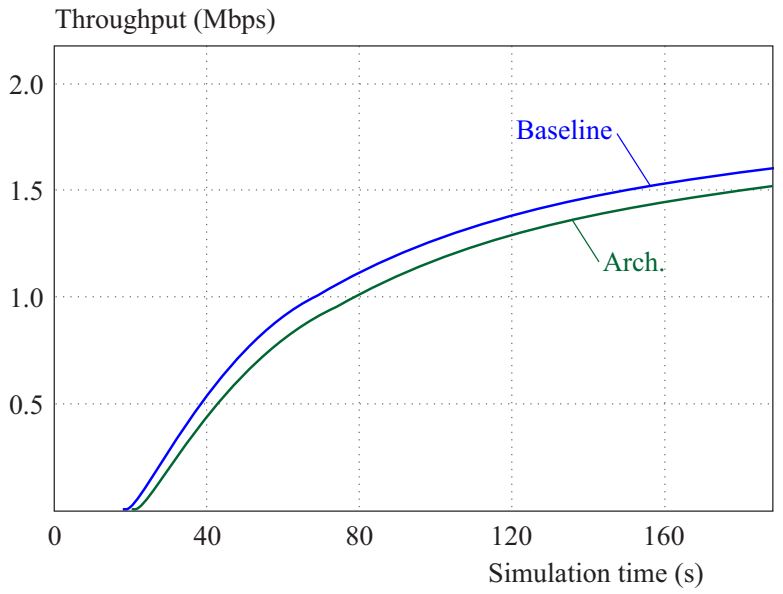

Fig. 15. Architecture Throughput comparison

\section{Conclusion}

In this paper, we presented our own security architecture for MANETs. We analyzed related work and then we described inner workings of our solution. We implemented it in a discrete event simulator and presented the results. Compared to existing solution, we noticed $3 \%$ worse throughput and $5 \%$ worse delay than analyzed solution [3]. Comparing the security of our solution, it is more thorough and advanced as any other analyzed solution. We provided architecture with secured routing protocol, PKI, Firewall concept and IPS. Nodes can have different privileges based on their trust level and if there is a suspicion about malicious behavior, those privileges can be reduced or revoked.

\section{Acknowledgements}

This work was partially supported by Eset Research Centre, research grant VEGA 1/0836/16 Methods and algorithms for effective and reliable delivery of multimedia content, and Research and Development Operational Programme for the project University Science Park of STU Bratislava, ITMS 26240220084, co-funded by the European Regional Development Fund.

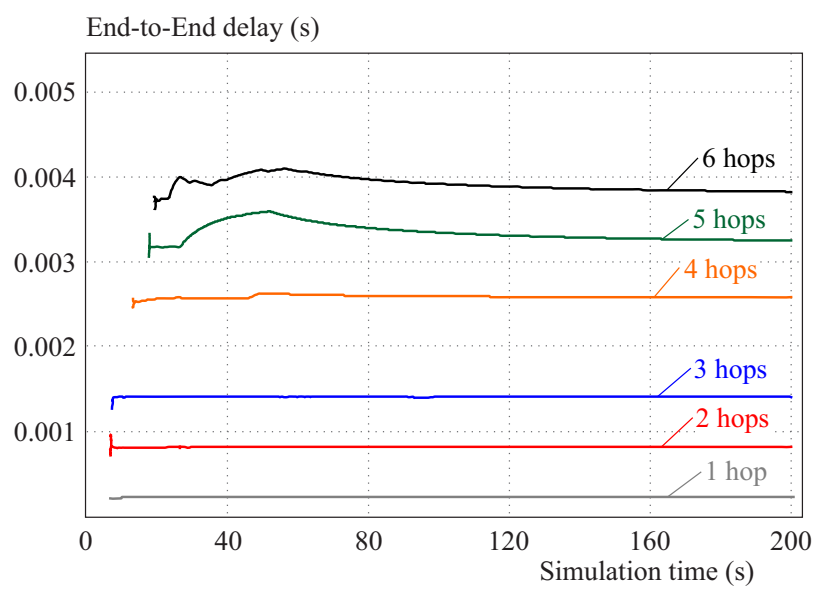

Fig. 14. Architecture latency

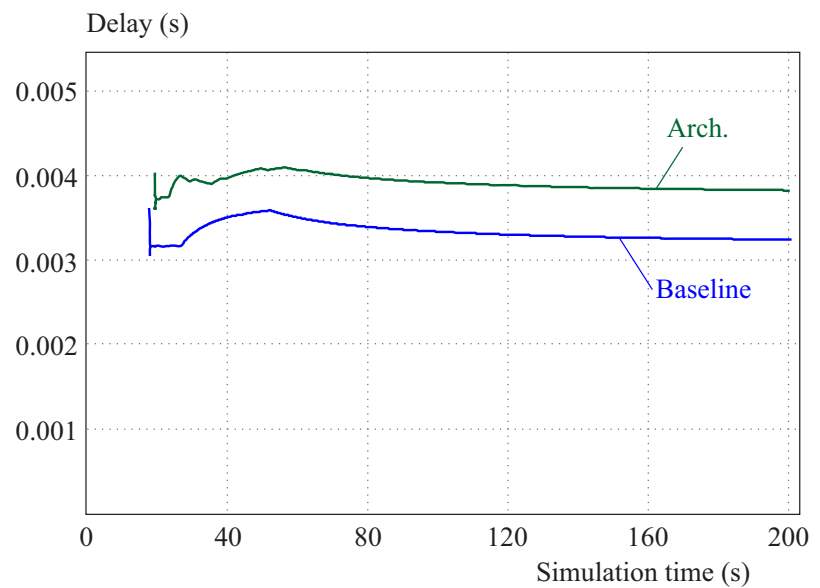

Fig. 16. Delay comparison

\section{REFERENCES}

[1] S. Akram, I. Zubair and M. Islam, "Fully distributed dynamically configurable firewall to resist DOS attacks MANET", rev. Networked Digital Technologies, 2009, NDT '09.First International Conference, Ostrava, 2009.

[2] M. K.A. D. ??? Alicherry, "Distributed Policy Enforcement Architecture for MANETs", Diploma work ???

[3] M. Alicherry, A. Keromytis and A. Stavrou, "Evaluating a collaborative defense architecture for MANETs", Rev. Internet Multimedia Services Architecture and Applications (IMSAA), 2009, IEEE International Conference, Bangalore, 2009.

[4] R. Boppana and X. Su, "On the Effectiveness of Monitoring for Intrusion Detection Mobile Ad Hoc Networks", 2011.

[5] A. Boukerche, K. El-Khatib, L. Xu and L. Korba, "SDAR: a secure distributed anonymous routing protocol for wireless and mobile ad hoc networks", 2004.

[6] A. Chaudhary, V. Tiwari and A. Kumar, "Design an anomaly based fuzzy intrusion detection system for packet dropping attack mobile ad hoc networks", Gurgaon, 2014.

[7] D. Ismail and M. Jaafar, "Mobile ad hoc network overview", Melaka, 2007.

[8] L. Jin, Z. Zhang, D. Lai and H. Zhou, "Implementing and Evaluating An Adaptive Secure Routing Protocol for Mobile Ad Hoc Network", Pomana, CA, 2006.

[9] I. Khalil, S. Bataineh, L. Qubajah and A. Khreishah, "Distributed secure routing protocol for Mobile Ad-Hoc Networks", Amman, 2013. 
[10] N. Noureldien, "A novel taxonomy of MANET attacks", Marrakech, 2015.

[11] L. Rajeswari, R. Annie and A. Kannan, "Enhanced intrusion detection techniques for mobile ad hoc networks", Tamil Nadu, 2007.

[12] S. Saha, R. Chaki and N. Chaki, "A New Reactive Secure Routing Protocol for Mobile Ad-Hoc Networks", Ostrava, 2008.

[13] B. Sun, L. Osborne, Y. Xiao and S. Guizani, "Intrusion detection techniques mobile ad hoc and wireless sensor networks",.

[14] S. Uyyala and D. Naik, "Anomaly based intrusion detection of packet dropping attacks mobile ad-hoc networks", Kanyakumari, 2014.

[15] B. Vaidya, D. Makrakis and H. Mouftah, "Provisioning secure on-demand routing protocol mobile ad hoc network", Kathmandu, 2011.

[16] P. Vilhan and L. Hudec, "Building Public Key Infrastructure for MANET with Help of BATMAN Advanced", Rev. Modelling Symposium (EMS), 2013 European, Manchester, 2013.

[17] N. V. Vinh, H. Jun and M.-K. Kim, "A Self-secure Routing Protocol for Large Mobile Ad hoc Networks", Singapore, 2007.

[18] H. Zhao and S. Bellovin, "High Performance Firewalls MANETs", Rev. Mobile Ad-hoc and Sensor Networks (MSN), 2010 Sixth International Conference, Hangzhou, 2010.

[19] L. Zhitang and S. Shudong, "A Secure Routing Protocol for Mobile Ad hoc Networks", Melbourne, Qld., 2007.

[20] D. Watkins and C. Scott, "Methodology for evaluating the effectiveness of intrusion detection tactical mobile ad-hoc networks", 2004.

[21] J. Filipek and L.Hudec "Distributed firewall using PKI mobile Ad Hoc networks", Proceedings of the 16-th International Conference on Computer Systems and Technologies, CompSysTech'15.

Received 22 March 2018

Jozef Filipek (Ing), currently pursuing his $\mathrm{PhD}$, Applied Informatics, Faculty of Informatics and Information Tech- nology, Slovak Technical University. In 2015 he received Ing diploma from Faculty of Informatics and Information Technologies, Slovak University of Technology, Bratislava. In the recent years he has focused his studies into networking technologies and have worked on networking positions within companies like ATT and DXC. He heas obtained CCNP and CCDP certificates and currently is working on acquiring CCIE certification. He is author of the several scientific papers published in journals and proceedings of the conferences.

Ladislav Hudec (doc, Ing, CSc), currently Associate Professor of Computer Science and Engineering, Deputy Director of the Institute of Computer Engineering and Applied Informatics, Faculty of Informatics and Information Technology, Slovak Technical University. In 1974 he received Ing diploma with summa cum laude in electronics from Faculty of Nuclear Sciences and Physical Engineering, Czech Technical University, Prague, in 1985 he received CSc degree ( PhD) in Computer Machinery from the Faculty of Electrical Engineering, Slovak Technical University, Bratislava, in 1989 he was appointed Associate Professor. Since 1974 he is with the Slovak Technical University. During the period 1992-1993 he served as Director of the SARC - Centre for Advancement, Science and Technology, Bratislava. He is author or co-author over 60 scientific papers published in journals and proceedings of the conferences and over 80 technical papers in the field of fault tolerant computing, embedded systems, and computer security. He led over 90 research grants and industrial contracts. He reads lectures on Principles of information security, Security of information technologies and Internet security. Dr. Hudec is member of the Information System Audit and Control Association (ISACA), holder of the CISA license. During the period 1993-2010 he served as National Coordinator at the European Cooperation in Science and Technology (COST). 